\title{
Evaluación de la estrategia educativa para fortalecer el uso de la guía de tromboprofilaxis en un hospital de Colombia
}

\author{
Assessment of educational strategy to strengthen \\ the guide for the use of thromboprophylaxis in a \\ colombian hospital
}

\author{
Shirley Neireth Gallo, Luis Fernando Durán, Víctor Manuel Molano, \\ Nicolás Arturo Núñez • Neiva (Colombia)
}

\section{Resumen}

Objetivo: evaluar el uso de la guía de tromboprofilaxis realizado por los médicos que participaron en la estrategia educativa mediante la valoración de las historias clínicas de los pacientes adultos hospitalizados en el área médica o quirúrgica del Hospital Universitario Hernando Moncaleano Perdomo con el propósito de fortalecer las habilidades en la prevención del tromboembolismo venoso.

Material y métodos: estudio descriptivo, observacional, con medición antes y después, sin grupo control, al uso de la Guía de Tromboprofilaxis por parte de los médicos según las historias clínicas de los pacientes hospitalizados por más de 24 horas, mayores de 14 años que ingresaron a los servicios hospitalarios indicados y el criterio de exclusión fueron las historias de pacientes hospitalizados en los servicios: UCI, obstetricia, pediatría, psiquiatría y oftalmología. Se utilizó una estrategia de educación destinada a incrementar la adherencia del personal médico al uso de la Guía de Tromboprofilaxis adoptada de la $8^{\circ}$ Guía del ACCP (American College of Chest Physicians) en consenso con cada uno de los servicios médicos. Las variables utilizadas fueron de escala nominal dicotómica, razón por la cual se utilizó el cálculo de frecuencia y a partir de allí se calculó el porcentaje y la tabla de contingencia que permitió calcular la medida de riesgo relativo y prueba de hipótesis.

Resultados: en el Hospital Universitario Hernando Moncaleano Perdomo de la ciudad de Neiva se incrementó la prescripción de tromboprofilaxis por adherencia a la guía en $26 \%$ de diferencia entre la primera observación y la siguiente (pasó de 46 a 72\%) y sobrevino una reducción en la prescripción inadecuada de profilaxis en $26 \%$ entre la primera observación y la siguiente (pasó de 54 a $28 \%$ ).

Conclusión: la estrategia educativa implementada fue eficaz dado que aumentó la adherencia de los médicos a la guía de profilaxis. (Acta Med Colomb 2015; 40: 24-29).

Palabras clave: prevención secundaria, tromboprofilaxis, educación médica.

\footnotetext{
Abstract

Objective: to evaluate the guide for the use of thromboprophylaxis by doctors who participated in the educational strategy by assessing the medical records of hospitalized adult patients in the medical or surgical area of the Hernando Moncaleano Perdomo University Hospital in order to strengthen abilities in preventing venous thromboembolism.

Material and Methods: descriptive, observational study with measurement before and after the use of guide thromboprophylaxis, without control group, by physicians according to clinical records of patients older than 14 years hospitalized for more than 24 hours. The exclusion criteria were the stories of hospitalized patients in: ICU, obstetrics, pediatrics, psychiatry and ophthalmology services. An education strategy aimed at increasing the adhesion of the medical staff to use the "Guide thromboprophylaxis" adopted from the 8th Guide ACCP (American College of Chest Physicians) in
}

Dres. Shirley Neireth Gallo Díaz, Luis Fernando Durán Gutiérrez y Víctor Manuel Molano Trujillo: Especialistas en Medicina Interna, Departamento de Medicina Interna, Hospital Universitario Hernando Moncaleano Perdomo, Universidad Surcolombiana, Neiva; Dr Nicolás Arturo Núñez Gómez: Especialista en Psicología Clínica y de la Salud. Especialista en Estadística. Magíster en Filosofía. Doctor en Ciencias de la Salud. Profesor Adscrito al Departamento de Ciencias Clínicas, Facultad de Salud. Coordinador Grupo de Investigación Carlos Finlay. Neiva (Colombia).

Correspondencia. Dr. Nicolás Arturo Núñez Gómez. Neiva (Colombia).

E-mail: ninugo@usco.edu.co

Recibido: 15/III/2014 Aceptado: 23/II/2015 
agreement with each of the medical services. The variables used were of the dichotomous nominal scale, which is why the frequency calculation was used and thereafter the rate and contingency table was calculated, allowing to calculate the extent of relative risk and hypothesis testing.

Results: in the University Hospital Hernando Moncaleano Perdomo in the city of Neiva the prescription of guide thromboprophylaxis increased by adherence to the guide in $26 \%$ of difference between the first observation and the next (went from 46-72\%) and there was a reduction in inappropriate prescription of prophylaxis in $26 \%$ between the first observation and the next (went from $54-28 \%$ ).

Conclusion: the implemented educational strategy was effective given that it increased adherence of physicians to guide prophylaxis. (Acta Med Colomb 2015; 40: 24-29).

Keywords: secondary prevention, thromboprophylaxis, medical education

\section{Introducción}

La implementación de nuevas estrategias educativas que mejoren la adherencia al uso de las guías de manejo clínico en tromboprofilaxis, podría ayudar a disminuir la incidencia del Trombo Embolismo Venoso (TEV), con la consecuente disminución de los índices de mortalidad, morbilidad y de los periodos de estancia hospitalaria.

El $50 \%$ de los casos de TEV procede de pacientes hospitalizados, lo cual representa una causa importante de morbilidad y mortalidad hospitalaria, así como un alto costo para los servicios de salud. En EE.UU., el TEV muestra una incidencia anual aproximada de uno a tres casos por 1.000 habitantes por año (1). En ausencia de profilaxis adecuada, la incidencia de TEV varía entre 10-80\%, (2-4). En la población hospitalaria colombiana se encontró una incidencia de $7 \%$ (5).

El uso de tromboprofilaxis hospitalaria en la población mundial es de $56.7 \%$ y cuando se exigen como estándares de calidad los de la conferencia del Colegio Americano de Médicos del Tórax (American College of Chest Physicians, $A C C P$ ) desciende a $50.2 \%$ (2). En Colombia el $60 \%$ de los pacientes recibe algún tipo de tromboprofilaxis pero sólo es adecuada en $54.8 \%$ de los casos $(6,7)$.

Actualmente, los esfuerzos se orientan a diversificar las estrategias de prevención mediante el uso de herramientas como la educación, destinados a incrementar la adherencia del personal médico al uso de las guías, con el propósito de fortalecer el uso de medidas profilácticas las cuales han venido desarrollándose desde hace más de 15 años. Sin embargo, en los reportes de estudios se observa una baja adherencia a las guías, lo que se traduce en un uso inadecuado de la tromboprofilaxis en el paciente hospitalizado $(8,2)$.

El objetivo de esta investigación es evaluar el uso de la guía de tromboprofilaxis realizado por los médicos que participaron en la estrategia educativa mediante la valoración de las historias clínicas de los pacientes adultos hospitalizados en el área médica o quirúrgica del Hospital Universitario Hernando Moncaleano Perdomo con el propósito de fortalecer las habilidades en la prevención del tromboembolismo venoso.

Hipótesis $\mathbf{H}_{\mathbf{0}}$ : la implementación de una estrategia educativa para médicos que laboran en las áreas médicas o quirúrgicas del Hospital Universitario Hernando Moncaleano Perdomo no influye positivamente en el uso de la Guía de Tromboprofilaxis

\section{Material y métodos}

Estudio descriptivo, observacional, con medición antes y después, sin grupo control (9-12) al uso de la guía de tromboprofilaxis por parte de los médicos que participaron en la estrategia educativa mediante la valoración de las historias clínicas de los pacientes adultos hospitalizados.

El universo del estudio (13) fue constituido por 1322 historias clínicas de los pacientes mayores de 14 años que ingresaron a los servicios de medicina interna, cirugía general, ortopedia, urología, cirugía plástica, neurocirugía y ginecología en el Hospital Universitario de Neiva Hernando Moncaleano Perdomo, distribuidas así: 655 historias clínicas en el periodo del 15 al 31 de agosto de 2009 y 667 historias clínicas en el periodo del 15 de julio al 15 de agosto de 2011, elaboradas por los médicos que participaron en el proceso educativo. El marco muestral se conformó por las historias clínicas de los pacientes asignados y numerados de cada cama, en cada uno de los servicios enunciados. El elemento muestral fueron las historias clínicas de los pacientes hospitalizados por más de 24 horas, mayores de 14 años que ingresaron a los servicios hospitalarios indicados y el reclutamiento de las historias clínicas correspondió a historias de pacientes que se les asignó camas cuyo número de identificación correspondió a un número impar. El criterio de exclusión fueron las historias de pacientes hospitalizados en los servicios: UCI, obstetricia, pediatría, psiquiatría y oftalmología, en el periodo inicial (antes) se excluyeron 31 y en el final (después) 23. Teniendo en cuenta periodo de tiempo seleccionado y método de reclutamiento enunciado se conformó una muestra de 296 historias clínicas antes y 310 después de la intervención.

El instrumento de recolección de información fue una ficha donde se consignaba: primero, si el paciente necesita tromboprofilaxis según los criterios presentados en la guía. Segundo, se prescribió al paciente profilaxis farmacológica o mecánica y tercero, la tromboprofilaxis fue adecuada según el riesgo establecido de TEV, a dosis adecuada, fármaco adecuado según comorbilidades y considerando la presencia 
de contraindicaciones para su uso. El control de sesgo de información se realizó mediante entrenamiento a las personas que llenaron los formatos de recolección y luego una sola persona entrenada garantizaba la solidez de la información, mediante revisión de la información día a día.

Las variables observadas en el estudio fueron:

- Estrategia de educación destinada a incrementar la adherencia del personal médico al uso de la guía de tromboprofilaxis adoptada de la $8^{\circ}$ guía del ACCP en consenso con cada uno de los servicios médicos.

- Registro en historia clínica de la prescripción de las intervenciones que disminuyen la incidencia de TEV mediante profilaxis farmacológica o mecánica.

El estudio fue aprobado por el comité de ética, bioética e investigación del HUHMP en reunión de mayo 17 de 2009, acompañado por la dirección del HUHMP y orientado por el Departamento de Medicina Interna. La investigación fue clasificada sin riesgo (Art. 11), ya que fue documental y no se realizó ninguna intervención. Siendo de especial atención el pleno conocimiento por parte de todos los médicos del grupo de medicina interna de la naturaleza de la investigación, que se obtendría un beneficio para los pacientes expresada en la mejor calidad de atención médica y por ende el fortalecimiento de la seguridad del paciente, sin riesgo por ser documental y con libre elección para los médicos de participar en las actividades educativas programadas. Es de acotar que no se incluyó un grupo control en consideración a que se debe brindar la mejor atención disponible para cumplir con el precepto de beneficencia y de no maleficencia. No se adquirió ningún compromiso con las casas farmacéuticas y en la guía creada los medicamentos son mencionados por su nombre genérico. Se tuvieron en cuenta las reglamentaciones establecidas en la Resolución 008430 de 1993 emanada del Ministerio de la Protección Social y en particular aumentar la seguridad de la atención del paciente en el Hospital Hernando Moncaleano Perdomo.

Para el análisis de datos se partió del hecho que las variables utilizadas fueron de escala nominal dicotómica, razón por la cual se utilizó el cálculo de frecuencia y a partir de allí se calculó el porcentaje, la tabla de contingencia, que permitieron medir el riesgo relativo (RR), medida que para este caso particular busca determinar el efecto derivado de la exposición "Estrategia educativa implementada y orientada a los médicos para mejorar el uso del tromboprofilaxis" a un determinado factor en estudio, mejorar el uso de las guías de tromboprofilaxis que podría disminuir la incidencia del tromboembolismo venoso.

El resultado del RR (14) permite identificar la magnitud o fuerza de la asociación para comparar los resultados antesdespués. $\mathrm{Si}$, valor del $\mathrm{RR}=1$, entonces se considera para este caso que la implementación o no de una 'estrategia educativa orientada a los médicos para mejorar el uso del tromboprofilaxis' no afecta el uso correcta de ella, pero si RR $>1$ se considerará que la estrategia educativa implementada tuvo un efecto positivo sobre aumentar el uso de forma correcta. Se calculó además el respectivo intervalo de confianza de 95\% (IC 95\%) y el valor de P.

Para el procesamiento de los datos obtenidos se utilizó el programa Epiinfo, versión 3.5.1 del 2008. Las tablas se realizaron con el programa Microsoft Excel, 2007.

Tabla 1. Estrategia educativa implementada para mejorar el uso de tromboprofilaxis.

\begin{tabular}{|c|c|}
\hline Estrategia educativa & Tipo de estrategia \\
\hline $\begin{array}{l}\text { 1. Adopción de la guía de tromboprofilaxis del hospital utilizando las recomendaciones de la guía publicada por la revista CHEST } \\
\text { en junio de } 2008 \text {, sobre lo concluido en la octava conferencia en terapia trombolítica y antitrombótica. Estas recomendaciones } \\
\text { fueron discutidas con cada grupo de especialistas de los diferentes servicios. Realizado en el primer semestre de } 2010 \text {. }\end{array}$ & $\begin{array}{l}\text { Estrategia educativa (adopción de la guía } \\
\text { en consenso con los médicos especialistas). }\end{array}$ \\
\hline $\begin{array}{l}\text { 2. Diseño de herramienta para facilitar el acceso a la guía, para ello se diseñó y se imprimieron } 500 \text { copias. } \\
\text { Realizado en el primer semestre de } 2010 \text {. }\end{array}$ & Soporte de decisión \\
\hline $\begin{array}{l}\text { 3. Socialización de la guía } \\
\text { Se realizó un evento público al cual se invitó a todo el personal médico especialista que participó en la adopción de la guía, así } \\
\text { como también a los médicos residentes, generales e internos de la institución, con una participación de médicos especialistas: } \\
\text { Ginecólogos } 6 \text {, ortopedistas } 5 \text {, neurocirujanos } 5 \text {, cirujanos generales } 6 \text {, cirujanos plásticos } 3 \text {, urólogos } 3 \text {, ginecólogos } 5 \text {, } \\
\text { médicos internistas } 6 \text {, médicos internos } 40 \\
\text { Realizado en junio de } 2010 \text {. }\end{array}$ & Estrategia educativa \\
\hline $\begin{array}{l}\text { 4. Entrega de guías de bolsillo: durante la etapa de la socialización de la guía y asimismo en las actividades de educación médica } \\
\text { realizadas para fortalecer este proyecto. Realizado en junio de } 2010 \text {. }\end{array}$ & Soporte de decisión, proveer recordatorios \\
\hline $\begin{array}{l}\text { 5. Posters: Seis meses después de la socialización y entrega de las guías de profilaxis antitrombótica, se realizaron y ubicaron en } \\
\text { las áreas donde el personal médico realiza la evolución diaria unos posters con la misma información de las guías de bolsillo. } \\
\text { Realizado en el primer semestre de } 2011 \text {. }\end{array}$ & Proveer recordatorios \\
\hline $\begin{array}{l}\text { 6. Educación médica: seis meses después de la socialización de las guías, se realizaron reuniones informativas y educativas al } \\
\text { personal médico de mayor rotación dentro del hospital. Se les entregó la guía de bolsillo, e instruyó en su adecuada utilización } \\
\text { y se resolvieron las dudas existentes acerca de la profilaxis. Realizado en mayo de } 2011 \text {. }\end{array}$ & $\begin{array}{l}\text { Proveer educación médica, acciones de } \\
\text { auditoría y retroalimentación. }\end{array}$ \\
\hline
\end{tabular}




\section{Resultados}

Se adoptaron las recomendaciones de la 8a. guía del American College of ChestPhysicians ACCP (Conference on Antithrombotic and Thrombolytic Therapy. Prevention of Venous Thromboembolism). El principal objetivo de la guía fue identificar los pacientes adultos hospitalizados con riesgo de tromboembolismo venoso con el fin de fortalecer el adecuado uso de la profilaxis.

Se creó una guía de bolsillo cuyo objetivo fue fortalecer las habilidades médicas para realizar la estratificación del riesgo del TEV y en el uso de los medicamentos profilácticos. Además se crearon varios posters educativos para presentar la guía adoptada al personal médico, los cuales fueron expuestos en los servicios de medicina interna, cirugía, neurocirugía, ginecología, urgencias, salas de cirugía y pasillos. El objetivo era recordar la necesidad de realizar la tromboprofilaxis y brindar la información necesaria para la estratificación del riesgo de TEV en el paciente hospitalizado.

La educación médica se realizó en reuniones para la educación del personal de mayor rotación en la institución donde se les instruyó en el adecuado uso de la profilaxis siguiendo los lineamentos de la guía.

Se revisaron 606 historias, de la cuales 296 correspondieron al antes de la implementación de la estrategia educativa de intervención para motivar el uso de la guía de tromboprofilaxis por parte de los médicos en pacientes adultos hospitalizados, obteniéndose el siguiente resultado (Tabla 2).

En esta primera etapa se encontró que según la estratificación del riesgo, $57.4 \%$ de los pacientes necesitó profilaxis, $38.5 \%$ no la requería y $4.1 \%$ sí la necesitaba, pero estaba contraindicado. En la etapa antes de la intervención $63.8 \%$ necesitaba profilaxis, $34.5 \%$ no la requiere y $1.7 \%$ la necesita pero tiene alguna contraindicación.

El porcentaje de prescripción de profilaxis por parte de los médicos fue $47 \%$ antes de la intervención y $50.3 \%$ después de la intervención. Según la estratificación del riesgo, los médicos prescribieron un manejo adecuado antes de la intervención en $28.7 \%$ de los pacientes que requerían tromboprofilaxis y en $20.9 \%$ de los pacientes que no la requerían, y después de la intervención estos valores fueron de 44 y $28.7 \%$, respectivamente.

En la Tabla 3 se observa que la tromboprofilaxis adecuada pasó de $46 \%$ antes de la intervención, a $72 \%$ después de la
Tabla 2. Distribución de los casos según variables: diseño, ¿necesita tratamiento?, ¿recibió tratamiento? Y ¿el tratamiento fue correcto?

\begin{tabular}{|c|c|c|c|c|}
\hline Diseño & $\begin{array}{c}\text { ¿Necesita } \\
\text { tratamiento? }\end{array}$ & $\begin{array}{c}\text { ¿Recibió } \\
\text { tratamiento? }\end{array}$ & $\begin{array}{c}\text { ¿Fue correcto } \\
\text { el tratamiento? }\end{array}$ & Frecuencia \\
\hline \multirow[t]{12}{*}{ Antes } & \multirow[t]{4}{*}{ Sí } & \multirow[t]{2}{*}{ Sí } & Sí & 63 \\
\hline & & & No & 22 \\
\hline & & \multirow[t]{2}{*}{ No } & Sí & 0 \\
\hline & & & No & 85 \\
\hline & \multirow[t]{4}{*}{ No } & \multirow[t]{2}{*}{ Sí } & Sí & 0 \\
\hline & & & No & 52 \\
\hline & & \multirow[t]{2}{*}{ No } & Sí & 62 \\
\hline & & & No & 0 \\
\hline & \multirow{4}{*}{$\begin{array}{c}\text { Sí pero } \\
\text { contraindicado }\end{array}$} & \multirow[t]{2}{*}{ Sí } & Sí & 0 \\
\hline & & & No & 2 \\
\hline & & \multirow[t]{2}{*}{ No } & Sí & 10 \\
\hline & & & No & 0 \\
\hline \multirow[t]{12}{*}{ Después } & \multirow[t]{4}{*}{ Sí } & \multirow[t]{2}{*}{ Sí } & Sí & 130 \\
\hline & & & No & 7 \\
\hline & & \multirow[t]{2}{*}{ No } & Sí & 0 \\
\hline & & & No & 61 \\
\hline & \multirow[t]{4}{*}{ No } & \multirow[t]{2}{*}{ Sí } & Sí & 0 \\
\hline & & & No & 18 \\
\hline & & \multirow[t]{2}{*}{ No } & Sí & 89 \\
\hline & & & No & 0 \\
\hline & \multirow{4}{*}{$\begin{array}{c}\text { Sí pero } \\
\text { contraindicado }\end{array}$} & \multirow[t]{2}{*}{ Sí } & Sí & 0 \\
\hline & & & No & 1 \\
\hline & & \multirow[t]{2}{*}{ No } & Sí & 4 \\
\hline & & & No & 0 \\
\hline \multicolumn{4}{|l|}{ Total } & 606 \\
\hline
\end{tabular}

Tabla 3. Tabla de contingencia del diseño de la investigación versus tromboprofilaxis adecuada o inadecuada.

\begin{tabular}{|l|c|c|}
\hline & Antes & Después \\
\hline TPF adecuada & $135(46 \%)$ & $223(72 \%)$ \\
TPF inadecuada & $161(54 \%)$ & $87(28 \%)$ \\
\hline Total: 606 & $\mathbf{2 9 6}$ & $\mathbf{3 1 0}$ \\
\hline \multicolumn{2}{|c|}{ R-R 1.58 (IC95\% 1.37-1.82), P < 0.001} \\
\hline TPF: Tromboprofilaxis. R-R: Riesgo relativo. IC: Intervalo de confianza. P= P_valor. \\
\hline
\end{tabular}

intervención y la inadecuada, de 54-28\%, respectivamente, con una diferencia estadísticamente significativa antes y después de la intervención, para mejorar el uso de la profilaxis adecuada y disminuir su uso inadecuado RR 1.58 [IC95\% 1.37-1.82], $\mathrm{p}<0.001$.

La Tabla 4 permite observar el porcentaje de prescripción y uso adecuado de tromboprofilaxis, encontrándose antes de la intervención el $21.2 \%$ de los pacientes que recibieron

Tabla 4. Tabla de contingencia del diseño de la investigación versus los que prescribieron o no tromboprofilaxis y la determinación de la prescripción adecuada de la misma.

\begin{tabular}{|c|c|c|c|c|c|}
\hline & Antes & Después & $\mathbf{R}-\mathbf{R}$ & IC & $\mathbf{P}$ \\
\hline Se prescribe TPF adecuada & $63(21.2 \%)$ & $130(42 \%)$ & 1.19 & $1.05-1.36$ & 0.01 \\
\hline Se prescribe TPF de manera inadecuada & $76(25.6 \%)$ & $26(8.3 \%)$ & 0.33 & $0.22-0.50$ & 0.000 \\
\hline No se prescribe TPF y fue adecuado $(*)$ & $72(24.3 \%)$ & $93(30 \%)$ & 1.23 & $0.95-1.6$ & 0.11 \\
\hline No se prescribe TPF y fue inadecuado & $85(28.7 \%)$ & $61(19.6 \%)$ & 0.69 & $0.51-0.91$ & 0.009 \\
\hline Total: 606 & 296 & 310 & & & \\
\hline
\end{tabular}


profilaxis fue adecuada versus el $42 \%$ después de la intervención R-R de 1.19 [IC95\% 1.05-1.36], $(\mathrm{p}=0.01)$.

En cuanto a los pacientes que, según la estratificación del riesgo, no requerían profilaxis y por lo tanto fue correcto que no se les administrara, fue $24.3 \%$ antes de la intervención y $30 \%$ después de la intervención ( $\mathrm{p}=0.11$ ).

\section{Discusión}

Se adoptó la guía de tromboprofilaxis del $8^{\circ}$ American College of Chest Physicians similar a lo realizado en otros estudios, en los que las recomendaciones del ACCP se consideraron como fuente principal y se hicieron adaptaciones de esta guía como la realizada en la Clínica El Country, al igual que en otras instituciones en el mundo (15-18) o se establecieron guías institucionales propias (19).

Desde hace más de quince años se elaboran guías y protocolos de manejo por diferentes organizaciones. Ejemplo de éstas son las publicadas por el American College of Chest Physicians (ACCP), el National Institute for Health and Clinical Excellence (NICE) y el Scottish Intercollegiate Guidelines Network, entre otras; pero la sola disponibilidad de las guías no ha tenido impacto en el adecuado uso de profilaxis.

La creación de la guía de bolsillo y de los posters mejoró las habilidades de los médicos para la estratificación del riesgo de los pacientes y aumentó el uso adecuado de la profilaxis. Otros estudios también han mostrado que la utilización de escalas de estratificación de riesgo, disponibles en las historias clínicas, son estrategias útiles para mejorar la adherencia, así como la distribución de posters ubicados en los sitios de prescripción (ídem). Otra estrategia similar y útil son las escalas de estratificación creadas para sistemas computarizados que muestran alarmas electrónicas, la cual fue una herramienta usada para evaluar el riesgo de TEV (20).

Se llevaron a cabo actividades de educación médica para fortalecer la divulgación y uso de la guía adoptada, lo que contribuyó a mejorar la adherencia por parte del personal médico al adecuado uso de la profilaxis antitrombótica. Una estrategia similar implementada en el estudio de Rocha y colaboradores en cuatro hospitales de Salvador (Brasil), tuvo en cuenta la importancia de brindar educación médica en reuniones donde además se entregaban folletos, algoritmos y posters (19).

La prevalencia del uso de tromboprofilaxis hospitalaria encontrada antes de la intervención y después de la intervención es entre 47-50.3\%, respectivamente, lo cual es similar al porcentaje encontrado en la población mundial reportado en los estudios en un rango de $50-60 \%(21,22)$ y en Colombia de $60 \%$, según los resultados para el estudio IMPROVE en nuestro país (23). Estos datos sólo hablan del uso general de la profilaxis por parte de los médicos sin tener en cuentas las exigencias de la guía adoptada.

Se observó un aumento en el uso adecuado de la profilaxis de $21 \%$, pasando el valor de $\mathrm{p}=0.01$ de $21.2 \%$ antes de la intervención al $42 \%$ después de la intervención. La "pro- filaxis adecuada" a nivel mundial es de aproximadamente $50 \%$ (16) y en Colombia, según el estudio de Velásquez y colaboradores realizado en la Clínica El Country es de $34.5 \%$ (7).

En los casos en que el médico prescribió la profilaxis, pero la utilizó en forma inadecuada antes de la intervención, fue de $25.6 \%$ y disminuyó después de la intervención a $8.3 \% \mathrm{p}=0.000$, lo que representa una disminución de $17 \%$. En este estudio la causa más frecuente de error fue la prescripción de profilaxis farmacológica a dosis mayores a las recomendadas.

Los estudios de Velásquez et al. Aquino Carneiro H, Rocha et al. y Hernández et al. muestran como causa frecuente de profilaxis inapropiada la utilización de los medicamentos profilácticos a dosis y frecuencia errada; por lo tanto es importante que se haya logrado mejorar en ese aspecto ( 7 , $15,16,24)$. El uso inadecuado de la medicación, ya sea por dosis mayores o menores a las recomendadas, aumenta los costos hospitalarios y expone a los pacientes a un riesgo innecesario de sangrado o trombosis, respectivamente.

En el grupo de pacientes que según la estratificación del riesgo corresponde a riesgo bajo y por tanto no requieren el uso de medidas profilácticas, en los cuales la medida correcta es NO prescribir profilaxis, pasó de $24.3 \%$ antes de la intervención a $30 \%$ después de la intervención ( $\mathrm{p}=0.11$ ), lo que disminuye los costos y la posibilidad de efectos adversos previsibles e innecesarios secundarios al uso de profilaxis. Estos hallazgos se comparten con el estudio realizado por Hernández et al. en Colombia. Es importante entonces dirigir los esfuerzos a mejorar las tasas de profilaxis, disminuyendo simultáneamente la profilaxis innecesaria (5).

La adopción y diseminación de la guía sumado a la utilización de una estrategia multifacética que incluyó actividades de educación, entrega de escalas de estratificación y la diseminación de posters recordatorios, fueron determinantes en la conducta médica para mejorar la prescripción adecuada de la profilaxis tromboembólica, pasando de 46-72\% al final de la intervención.

Se obtuvieron resultados similares en los estudios de Scaglione y colaboradores, donde a través de las mismas estrategias educativas se obtuvo un aumento en el uso de tromboprofilaxis de $25-41 \%$ en paciente médicos y de 64 $97 \%$ en pacientes quirúrgicos (25). Al-tawfiq y colaboradores utilizaron la educación médica como método principal, acompañado de múltiples intervenciones de retroalimentación cuando se identificaban errores en la prescripción o estratificación del riesgo, pasando de un uso de profilaxis de $63-100 \%$ al terminar el estudio (26).

Las limitaciones encontradas en el estudio fueron la no medición del impacto de la guía y el no seguimiento de los pacientes durante un tiempo prolongado, para poder determinar cuántos casos de TEV se presentaron por el uso inadecuado de la profilaxis y cuántos se previnieron gracias al uso de la misma. Además, el mismo diseño de investigación limita la validez externa de los resultados debido a 
múltiples y complejas del ambiente que pueden afectar la relación causal de los resultados.

\section{Conclusión}

La estrategia educativa implementada en el Hospital Universitario Hernando Moncaleano Perdomo fue eficaz, dado que aumentó la adherencia de los médicos a la guía de profilaxis adoptada, lo cual se logró a través de la adopción de una guía basada en evidencia científica y la realización de actividades de educación médica que llevó a un aumento del uso y el "adecuado uso" de la profilaxis por parte de los médicos de la institución y fortaleció las habilidades de los médicos que les permite prevenir el tromboembolismo venoso al aumentar la prescripción adecuada de tromboprofilaxis.

\section{Agradecimientos}

Los autores expresan su agradecimiento a la dirección del Hospital Universitario Hernando Moncaleano Perdomo, al Departamento de Medicina Interna, a la Facultad de Ciencias de la Salud de la Universidad Surcolombiana, a los Laboratorios Pfizer de Colombia S.A. por su apoyo en la creación de la clínica de anticoagulación del Hospital Hernando Moncaleano Perdomo. Además el apoyo del profesor de la Universidad Surcolombiana, Giovani Durán. Mendeley Desktop por permitir utilizar su plataforma para manejar las referencias bibliográficas.

\section{Referencias}

1. Rosendaal, F., and Biller, H. Trombosisvenosa. IN. Fauci, A., Braunwald, E., Kasper, D., Hauser, S. Longo, D., Jameson, L. and Loscalzo, J. Harrison Principios de Medicina Interna Edic 17. Capítulo 11, página 731-35.

2. Geerts, W., Bergqvist, D, and Pineo, G., et al. Prevention of venous thromboembolism: American College of Chest Physicians Evidence-Based Clinical Practice Guidelines (8th Edition). Chest 2008; 133(6 Suppl): 381S-453S.

3. Pineo, G. Prevention of venous thromboembolic disease in surgical patient. Uptodate 2012. [acceso 4 de junio de 2012]. Disponible en: http://www.uptodate. com

4. Kahn, S., Lim, W., Dunn, A., Cushman, M., Dentali, F. Prevention of VTE in Nonsurgical Patients. Antithrombotic Therapy and Prevention of Thrombosis, 9th ed: American College of Chest Physicians Evidence-Based Clinical Practice Guidelines. 141/2/ February, 2012 Supplement.

5. Dennis, R., De Arboleda M., Rodríguez M., Salazar M., Posada P. A nombre de GRITE. Estudio nacional sobre tromboembolismo venoso en población hospitalaria en Colombia. Acta Med Colomb 1996; 21: 55-63.

6. Dennis, R., Roa, J., Villadiego, J., Méndez, F., Vieda, E. y Restrepo, H. Profilaxis de la tromboembolia venosa en pacientes colombianos de tratamiento médico o quirúrgico: resultados para Colombia del estudio ENDORSE. Biomedica 2011; 31: $200-8$

7. Velásquez, J., Herrán, S. y Mera, I. Uso de tromboprofilaxis en el paciente hospitalizado en la Clínica del Country, Un análisis de la adherencia a la guía institucional. Acta Med Colomb 2010; 35: 8-14.

8. Al-Tawfiq J. and Saadeh B. Improving adherence to venous thromoembolism prophylaxis using multiple interventions. Ann Thorac Med 2011; 6: 82-4.
9. Manterola, C. Estudios observacionales. los diseños utilizados con mayor frecuencia en investigación clínica. Rev Med Clin Condes 2009; 20(4): 539 - 548 http://www.clinicalascondes.cl/Dev_CLC/media/Imagenes/PDF\%20revista\%20 m\%C3\%A9dica/2009/4\%20julio/539_ESTUDIOS_OBSERVACIONALES-21. pdf.

10. Glasser, S., and Howard, G. Clinical Trial Design Issues: At Least 10 Things You Should Look For in Clinical Trials. J. Clin. Pharmacol. 2006; 46: 1106.

11. Hernández, R., Fernández, C., y Baptista, M. Metodología de la investigación. México, Mc Graw Hill, 2006.

12. Polit, D. y Hungler, B. Investigación científica en ciencias de la salud. $6^{\mathrm{a}}$ ed México: McGraw-Hill Interamericana, 2000

13. Bello, León Darío. Estadística como apoyo a la Investigación. Editorial L.Vieco e Hijas Ltda., abril de 2005.

14. Escuela Nacional de Sanidad (ENS). Instituto de Salud Carlos III-Ministerio de Ciencia e Innovación. Miguel Ángel Royo Bordonada, Javier Damián Moreno, "Método epidemiológico". Madrid: ENS-Instituto de Salud Carlos III, Octubre de 2009, http://www.isciii.es/ISCIII/es/contenidos/fd-publicaciones-isciii/fddocumentos/2009-0843_Manual_epidemiologico_ultimo_23-01-10.pdf, 2013.

15. Aquino, H., Pelegrineti, G., Oliveira, L. Avaliação da profilaxia do tromboembolismo venoso em hospital de grande porte. Rev Col Bras Cir 2010; 37(3): 204-210.

16. Rocha A., Braga, P., Ritt, G, et al. Inadequação de tromboprofilaxia venosa em pacientes clínicos hospitalizados. Rev Assoc Med Bras 2006; 52(6): 441-6.

17. Scaglione, L., Piobbici, M. et.al. Implementing guidelines for venous thromboembolism prophylaxis in a large Italian teaching hospital: lights and shadows Haematologica 2005; 90: 678-684.

18. Velásquez, J., Herrán, S., Mera, I. Uso de tromboprofilaxis en el paciente hospitalizado en la Clínica del Country, Un análisis de la adherencia a la guía institucional. Acta Med Colomb 2010; 35: 8-14.

19. Rocha, A., Paiva, E., Miranda De Araújo, D, et.al. Impacto de un programa para profilaxia de tromboembolismo venoso em pacientes clínicos. Rev Assoc Med Bras 2010; 56(2): 197-203.

20. Novis S., Havelka G., Ostrowski D., Levin B., Blum-Eisa L., Prystowsky J., Kibbe M. Prevention of thromboembolic events in surgical patients through the creation and implementation of a computerized risk assessment program. J Vasc Surg 2010; 51: 648-54.

Cohen,A., Tapson, V., and Bergmann, J. Venous thromboembolism risk and prophylaxis in the acute hospital care setting (ENDORSE study): a multinational crosssectional study. The Lancet Volume 371, Issue 9610, Pages 387-394, 2 February 20.

21. Victor F. Tapson, MD, FCCP; Herve« Decousus, MD; Mario Pini, MD; Beng H. Chong, MD, PhD; James B. Froehlich, MD, MPH. Venous Thromboembolism Prophylaxis in Acutely Ill Hospitalized Medical Patients*Findings From the IMPROVE. CHEST 2007; 132: 936-945

22. Dennis R, Acevedo J, et al. ¿Es apropiada la profilaxis actual del tromboembolismo venoso en pacientes médicos? Evaluación de desenlaces colombianos en el Registro Internacional sobre Prevención Médica del TEV (IMPROVE). Acta Med Colomb 2009; 34.

23. Hernández J, Dennis R, Londoño D, Gil J. Evaluación de concordancia entre estrategias de profilaxis de tromboembolismo venoso en paciente médico en un hospital universitario, Bogotá, D.C. 2007-2008. Acta Med Colomb 2009; 34: 4-10.

24. Scaglione, L., Piobbici, M., Pagano, E, Ballini, L., Tamponi, G. and Ciccone, G. Implementing guidelines for venous thromboembolism prophylaxis in a large Italian teaching hospital: lights and shadows Haematologic 2005; 90: 678-684.

25. Al-Tawfiq J, Saadeh B. Improving adherence to venous thromboembolism prophylaxis using multiple interventions. Annals of Thoracic Medicine. 2011; Vol 6 (2): $82-84$. 